\title{
Design and Implementation of Scene Roaming System Based on VR
}

\author{
Saihua Xu' ${ }^{\mathrm{a}}$, Yin Xiaohong, Xie Fangsen \\ Nanchang Institute of Science and Technology, Nanchang 330108, China \\ axusaihua@126.com
}

Keywords: VR; scene roaming system; computer graphics; information technology; panorama

\begin{abstract}
The biggest characteristic of VR virtual roaming is that the object being roamed exists objectively, but the form of roaming is virtual in a different place. At the same time, the making of roaming object is the real data based on object. Virtual reality technology is appeared at the end of twentieth Century a new comprehensive information technology, which combines digital image processing, computer graphics, multimedia technology, sensor technology and other information technology branch, which greatly promoted the development of the computer technology. The paper presents design and implementation of scene roaming system based on VR.
\end{abstract}

\section{Introduction}

VR panorama has broad applications, such as tourist attractions, hotels, construction and real estate; decoration exhibition. In architectural design, real estate or decoration can be accomplished by panoramic panorama technology. Not only make up for the shortcomings of a single point of view renderings, and three-dimensional animation to the economical and practical, the best choice as a designer.

The human in the pursuit of rapid economic growth, also requires a higher quality of life, the technology is playing a central role. Virtual simulation (VR) refers to a special environment generated by computer, people can through their "projection" to the environment. Use the special device and operation control of the environment, to achieve a specific purpose, which is to dominate this environment [1]. It has its immersive interactive (immersion), (interaction) and ideas (imagination), which can make people immersed, beyond its natural form, and, with the interactive performance of multidimensional the environment of information. Rapidly penetrated into all sectors of society, and has been used in computer aided design, engineering and scientific data visualization, 3D Geographic Information System (GIS), has been widely used in medical, gaming and entertainment.

In recent years, China's Internet penetration rate increased year by year, the Internet is going into the life and work of.CNNIC< report "people's investigation showed that at home and units in the proportion of Internet users in 2009 has been significantly improved, 83.2\% of Internet users choose the Internet at home, while $30.2 \%$ of people choose in units of the Internet network, the Internet as a tool for everyday the value is rising. Unlimited business opportunities in all walks of life came into being. With its sharp eyes have begun to explore their business opportunities on the Internet.

Virtual reality technology (Virtual Reality, referred to as VR) as a new media technology, its application areas including real estate planning, architecture and landscape design, Home Furnishing art design, experience education, medical simulation, military simulation, security monitoring, network simulation, traffic planning, cultural relics and ancient complex, virtual tourism, games and entertainment, and will gradually be involved to all walks of life, the full depth of the public daily life learning, become an integral part of the future digital life technology pillar.

Virtual reality, multimedia and network information technology for the protection of historical relics, provides new means and methods of restoration and research at home and abroad have been paid attention to. In early 1990s, the British Museum, the Metropolitan Museum and other large museum has realized the virtual roaming. In recent years, China with great development in digital 
cultural relics related areas, the Ministry of education established the "University Digital Museum Construction Engineering, Dunhuang Research Institute and Northwestern University jointly launched the" digital Dunhuang murals cooperative research ", the Imperial Palace Museum and Toppan Printing Company has developed a virtual the Imperial Palace in Beijing. The bid for the 2008 Olympic Games also put forward the" Virtual Olympic Museum "creative, has aroused great interest and concern of the International Olympic Committee, which host provides great help to get China Help.

The research on virtual reality technology in the collection shows the application of practical task, the use of virtualization, virtual exhibition cultural digital technology, improve the display rate and the display effect of cultural relics and cultural relics protection entities, and further extended to break the constraints of time, the museum's collection, collection, exhibition and cultural dissemination function.

\section{Interactive roaming system based on VR}

According to the connotation and essential characteristics of virtual reality technology, it can be seen that its research and development is a relatively high technical requirements, it needs a corresponding software and hardware system environment to be matched. In addition to the perfect virtual reality software development platform and three-dimensional image processing system, according to the technical characteristics of virtual reality [2]. The system also requires a highly lifelike three-dimensional immersion, which is mainly realized by three-dimensional hearing, three-dimensional tactile or force sense and visual environment with high immersion. Stereo hearing is usually realized by three-dimensional surround stereo sound system, while highly immersive visual environment is usually realized by large screen stereoscopic projection display system.

In addition, according to the technical characteristics of virtual reality, real-time interaction is the soul of virtual reality technology, which is different from other traditional media technology in essence. In virtual reality system, this kind of interaction is usually realized by virtual reality interactive device, and finally a complete virtual reality realization system is formed.

This article from the modeling and rendering of 3D MAX baking technology to 3D campus roaming system using mature VRP-BUILDER virtual reality editor module to build a two development.

The development of 3D and the method of 3D simulation roaming system based on VRP technology, Wuzhou University (North) to build the virtual scene, automatic roaming, manual roaming Campus navigation path, view the scenery of the campus, the campus information query, climate effect, dynamic effect of various entities, and do a detailed route according to the collision detection. At the same time according to the characteristics of 3D simulation roaming, roaming in the automatic and manual roaming process, based on the existing scene as the foundation, through the video, pictures, music. To the virtual reality system; provide convenience for the need to understand the Wuzhou University campus geographic information users [3].

$$
w_{j}(k) \equiv \sum_{l=0}^{L_{j}-1} h_{j, l} x_{i}((k-l) \bmod N), \quad k=0,1, \cdots, N-1
$$

The modeling method of Polygon+NURBS advanced modeling, each model using simplified model to the three-dimensional virtual campus architecture; using Bitmap bitmap +UVW Mapping mapping, VRAY real scene rendering method for reduction of the campus; using Max-for-VRP derived plug-in model into VRP-BUILDER virtual reality editor module, adding collision detection algorithm, VRP realize man-machine command line scripts the interactive function, to ensure the practicality of the system; the use of walking camera, dynamic roaming increase real 3D performance, multi angle view school environment; running from virtual reality editor module is derived for the EXE portable can run the executable file system.

According to the real terrain data is used for terrain generation of a class of the most, at present 
most of the digital terrain model (Digital Terrain Model, DTM) to generate DTM data, by the sampling elevation in the grid map the value composition corresponding to the remote sensing image data captured texture plane or satellite.

The texture image is mapped to the corresponding part in the reconstruction of terrain surface. Terrain rendering algorithm is simple, the DTM cell transformation of 4 adjacent grid points defined into 2 dimensional space of the triangle, then the optic internal area of pyramidal all such triangles sent to the graphics pipeline drawing.

This algorithm can also be the image texture data to the highest resolution mapped to the corresponding polygon, but this is a very inefficient, because in general, triangle and remote sensing images The number of physical pixels is very large, and each individual triangle projection to the image space is very small, and a lot of texture pixels may be compressed to a pixel in the image, so that the effect is negligible [4]. Therefore, if directly generated by DTM terrain, even in high performance graphics hardware platform on real-time rendering, it is almost impossible, usually needs to be simplified to DTM. Data simplification methods will be discussed in detail in the next chapter.

The biggest characteristic of this kind of virtual roaming is that the object being roamed is already objective and real, but the form of roaming is only fictitious in different places and at the same time. Roaming object making is real data based on object. It creates a virtual information environment in multidimensional information space, which can make users feel immersive and have perfect interaction ability with environment. And it helps to enlighten the idea that VR has not only been focused on computer graphics, it has been involved in a wider range of fields, such as videoconferencing, network technology and distributed computing technology. Virtual reality technology has become an important means of new product design and development.

\section{Design of 3D VR scene roaming system}

The virtual scene simulation technology is regarded as an important branch of virtual scene technology. Computer technology, image processing and graphics generation technology, multimedia technology, information synthesis technology, the integrated use of display technology and other high technology, its components include simulation modeling technology, animation technology and real-time visual technology at present domestic virtual scene technology market has not yet substantial development, but also has begun to take shape [5].

The United States is in the leading position in the field, the basic research mainly focuses on perception, user interface, the four aspects of software and hardware. NASA (NASA) research focused on real time simulation of space station operation, they used a lot for the cockpit flight simulation technology [6]. The University of North Carolina (UNC) the computer department developed a help users in complex visual parallel processing system for real-time dynamic display of building landscape.

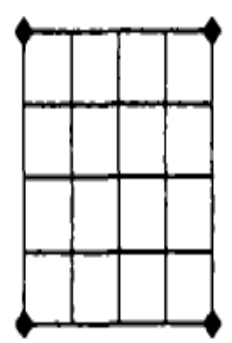

(a)

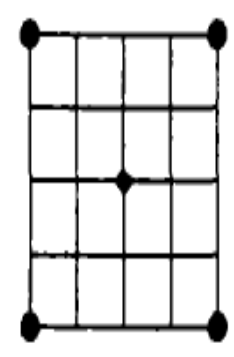

(b)

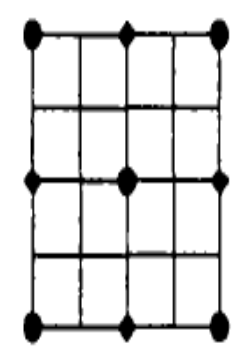

(c)

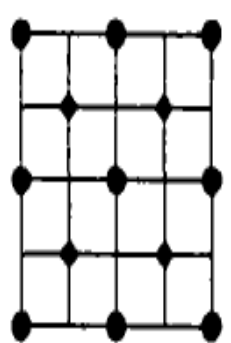

(d)

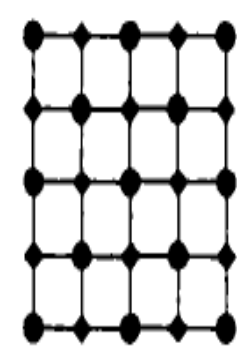

(e)

Figure1. vehicle real-time 3D visual simulation and virtual environment

Massachusetts Institute of Technology (Mrr) in 1985 to set up a media lab, a man named BOLIO 
test environment for different graphic simulation experiment. University of Washington Washington Technology Center (HIT Lab) Interface Technology Laboratory of feeling, perception, cognition and motion control ability of.DaveSimS et al developed a virtual reality model to see how the system operates retreat in Illinois.

The State University developed in vehicle design, system realization, distributed virtual remote collaboration support in different countries; different regions of the engineers can design through real-time collaboration computer network. George Mason University developed in a dynamic virtual environment in real-time fluid simulation system [7]. The California Graduate School of Naval Research Laboratory of NPS visualization the work in the virtual environment navigation and simulation.

In order to achieve IEEE in distributed interactive simulation (Dls) network protocol under the support of the vehicle real-time 3D visual simulation and virtual environment. The Wright Patterson Air Force Base "3D image and Computer Graphics Lab" is S on GI4D/400 workstation built space satellite the virtual environment to simulate near space and describe the 3D graphical model of satellite earth's orbit and the running state of the simulation The information of the simulation object is more fully [8].

Virtual reality (Virtual Reality VR) technology is appeared at the end of twentieth Century a new comprehensive information technology, which combines digital image processing, computer graphics, multimedia technology, sensor technology and other information technology branch, which greatly promoted the development of computer technology.

The virtual technology of virtual reality technology (King) (such as virtual tour entity and Virtual Museum) virtual environment (landscape) technology (such as the restoration of generation Epang palace, Old Summer Palace has lost the building, construction has not yet been discovered Mausoleum of the First Qin Emperor) two categories. Application of virtual reality technology and cross field is very extensive. At present the successful use of the field of battlefield virtual reality technology the virtual reality simulation environment, combat command, aircraft, ship, vehicle virtual reality driving training, aircraft, missiles, ships and cars (virtual manufacturing virtual design system, including virtual reality construction) Display and visit of buildings, virtual reality surgery training, virtual reality game, virtual reality, film and television art, etc. so we can see that VR technology has strong market demand and technology drive [9].

The construction of the integrated innovation of virtual reality system to realize the reconstruction of the product can be applied research and innovation training platform based on the overall goal is through the use of scientific, reasonable configuration, virtual laboratory system, the establishment of a virtual laboratory environment with the participants feel personally on the scene and real-time interactive capabilities, which will enhance the level of scientific research and teaching environment to a with the level of technological innovation platform. After the completion of the "integrated innovation based on reconfigurable product system virtual reality application of innovative research and training platform" should be a set of teaching, scientific research and demonstration functions, with immersive display and real-time interaction as the main function of the virtual reality laboratory environment and a new generation of digital media technology innovation platform.

\section{Design and implementation of scene roaming system based on VR}

The mathematical model of distribution of Brown motion to generate realistic scenes from random fractal, many nonlinear phenomena he can express effectively in nature, is so far the best to describe the real terrain. Then he is a generalization of the Brown movement. The algorithm is: random fractal terrain generation technology of fractal geometry and FMB based on the method, used a Poisson step method (poissonfaulting), Fu Liye filter (fourierfiltersng), the midpoint displacement method (midpointdisplaeement), successive random additions (Suc.essiverandomadditions) and band limited noise accumulation method (summingbandlimit.dnoises) and other five categories. Among them, the random midpoint displacement method is the most simple and classic that is a direct application of FBM. 
A one-dimensional random midpoint displacement method for his own thought is: the known vertex elevation (or attribute) line, the midpoint of the elevation ( For the ends of attributes) or height (or attribute) the average value plus a random displacement, displacement of the two segment of the midpoint subdivision and recursive displacement, know that meet the resolution needed so far. The extension to the two-dimensional surface, according to the different pattern of the simulation can be divided into triangle grid simulation method, rectangular (square) grid simulation method, diamond square grid simulation method, parameter block grid simulation method, the thought and the one-dimensional similar. The square grid as an example the realization process of two-dimensional random midpoint displacement method.

Good computer games, not only can achieve the purpose of work alternately, eliminate fatigue, and cultivate intelligence sentiment and inspire imagination. Computer game show is mainly virtual editing script under artificial scene behavior changes. So the application and effect of the virtual building scene roaming technology in the field of play a decisive role.

The original delta game using a large number of indoor and outdoor architectural scenes, such as barracks, bunkers, tunnels, tower, armory, tower. Later the popular Quake, VR and other special police use the subway, train and ship building internal scene real-time strategy game has been more common. When this network game against the CS scene it is from 3D indoor and outdoor buildings. Even the sports games such as need for speed, FIFA, the stadium, bridge, tunnel and other buildings scene is also indispensable.

Battlefield virtual simulation and command simulation training have all kinds of virtues of virtual simulation technology, such as safety, repeatability, economy, difficulty of battlefield environment adjustability, convenient against simulation, easy to achieve various tactical settings and so on.

The virtual reality technology and multimedia technology, network technology is the application of computer technology in twenty-first Century three with the greatest development potential. Although the virtual reality technical difficulties still exist many unsolved theoretical problems have not yet overcome the impact on human life and work but also very little. However, it is foreseeable that in the near future, have a significant impact on the virtual reality technology is bound to human life and production.

\section{Summary}

The paper presents design and implementation of scene roaming system based on VR. Although all the countries have successfully developed some typical applications of virtual reality, but the application of high technology compared with other, is still in the initial stage of application development. Although it may not be able to clearly imagine, in the new century and new forms of popular virtual reality, but people can through the application of medium shape change the principle and extension of the field of medium main propagation characteristics, a reasonable conception of future scenarios.

\section{References}

[1] Wang Rui, the design and implementation of the money Xuelei.OpenSceneGraph 3D rendering engine. Beijing: Tsinghua University press, 2012.11.

[2] Zhu Danchen, song Guiling. The realization of computer and modernization of virtual museum system based on Unreal3 and 2013, 34:48-52.

[3] Duan Xinyu. The foundation of virtual reality and VRML programming. Beijing: Higher Education Press, 2014.3.

[4] Xiao Peng, Liu Gengdai, Xu Mingliang.OpenSceneGraph 3D rendering engine programming guide. Beijing: Tsinghua University press, 2012.

[5] Feng Yufen. Design and implementation of virtual cell roaming system based on Virtools. 
Computer simulation, 2015, 26 (6): 285-287.

[6] Jiang Xuezhi, Li Zhonghua. Research status of virtual reality technology at home and abroad.Journal of Liaoning University of Technology, 2016.

[7] Deng Zheng detailed translation of.OpenGL programming guide. Fourth edition. Beijing: people post and Telecommunications Press, 2015.

[8] Yuan Haibo, Liu Houquan, and so on. 3D interactive. Microcomputer information based on scene semantics in virtual museums, 2012, 25 (9-3): 175-177.

[9] Li Zhiwen, Han Xiaoling. Research status and future development of virtual reality technology and future development. Information technology and information technology (Human-Computer Interaction Edition) 2015 (3): 94 - 96. 